\title{
AN EXTENSION PROPERTY OF QUASIMÖBIUS MAPPINGS IN METRIC SPACES
}

\author{
Tiantian Guan, Manzi Huang and Xiantao Wang* \\ Shantou University, Department of Mathematics \\ Shantou, Guangdong 515063, P. R. China; 17ttguan@stu.edu.cn \\ Hunan Normal University, School of Mathematics and Statistics, MOE-LCSM \\ Changsha, Hunan 410081, P. R. China, and \\ Qufu Normal University, School of Mathematical Science \\ Qufu, Shandong 273165, P. R. China; mzhuang@hunnu.edu.cn \\ Hunan Normal University, School of Mathematics and Statistics, MOE-LCSM \\ Changsha, Hunan 410081, P. R. China; xtwang@hunny.edu.cn
}

\begin{abstract}
In 1991, Väisälä discussed the extension property of quasisymmetric mappings in Banach spaces. In 2009, Haïssinsky got an extension property of quasisymmetric mappings in metric spaces. The purpose of this paper is to establish an extension property of quasimöbius mappings in metric spaces.
\end{abstract}

\section{Introduction}

\subsection{Extension of quasisymmetric mappings.}

Definition 1.1. Suppose that $(X, d)$ and $\left(Y, d_{1}\right)$ are metric spaces and $\eta:[0,+\infty)$ $\rightarrow[0,+\infty)$ is a homeomorphism with $\eta(0)=0$. A homeomorphism $f:(X, d) \rightarrow$ $\left(Y, d_{1}\right)$ is called $\eta$-quasisymmetric, briefly $\eta$-QS, if $d(a, x) \leq t d(x, b)$ implies

$$
d_{1}\left(a^{\prime}, x^{\prime}\right) \leq \eta(t) d_{1}\left(x^{\prime}, b^{\prime}\right)
$$

for any points $a, x, b$ in $X$ and any number $t \geq 0$. Here and hereafter, the primes always stand for the images of the points under the mappings. For example, $a^{\prime}=$ $f(a)$.

Quasisymmetric mappings originate from the work of Beurling and Ahlfors [4], who defined them as the boundary values of quasiconformal self-mappings of the upper half-plane onto the real line. The general definition of quasisymmetric mappings, i.e., Definition 1.1, is due to Tukia and Väisälä [10]. Since its appearance, the concept of quasisymmetric mappings has been studied by numerous authors. See, for example, [3, 8] for the properties of this class of mappings. In 1991, Väisälä discussed the extension property of quasisymmetric mappings in the setting of Banach spaces and proved the following result.

Theorem A. [13, Theorem 7.39] Suppose that $f: E \rightarrow E_{1}$ is a homeomorphism and $E=A \cup B$ such that the restrictions $\left.f\right|_{A}$ and $\left.f\right|_{B}$ are $\eta$-quasisymmetric, where both $E$ and $E_{1}$ are Banach spaces with dimension at least 2, and $A$ and $B$ are subsets of $E$. Then $f$ is $\eta_{1}$-quasisymmetric, where $\eta_{1}$ depends only on $\eta$.

In [7], the author considered the extension property of quasisymmetric mappings in metric spaces. The obtained result is as follows.

https://doi.org/10.5186/aasfm.2020.4501

2010 Mathematics Subject Classification: Primary 30F45; Secondary 30C65, 30L05, 30L10.

Key words: Quasimöbius mapping, quasiconvexity, metric space.

*Corresponding author. 
Theorem B. [7, Theorem 3.1] Suppose $X=X_{1} \cup X_{2}$ and $Y=Y_{1} \cup Y_{2}$ are metric spaces with $\angle\left(X_{1}, X_{2}\right)>0$ and $\angle\left(Y_{1}, Y_{2}\right)>0$. Assume that $X_{1} \cap X_{2}$ and $Y_{1} \cap Y_{2}$ are $\tau$-uniformly perfect such that $\operatorname{diam}\left(X_{1} \cap X_{2}\right) \geq q \operatorname{diam} X_{1}$ for some $q \in(0,1)$, where "diam" means "diameter". If $f: X \rightarrow Y$ is a homeomorphism such that for each $j \in\{1,2\}, f\left(X_{j}\right)=Y_{j}$ and $\left.f\right|_{X_{j}}$ is $\eta$-quasisymmetric, then $f$ is globally $\eta_{1^{-}}$ quasisymmetric, where $\eta_{1}$ depends only on $\eta, \tau$, $q$, together with the angles $\angle\left(X_{1}, X_{2}\right)$ and $\angle\left(Y_{1}, Y_{2}\right)$.

See Section 2 for the definitions of the angle $\angle\left(X_{1}, X_{2}\right)$ and the uniform perfectness. We remark that the assumption on the uniform perfectness of $Y_{1} \cap Y_{2}$ in Theorem B is redundant since the concept of uniform perfectness is an invariant under quasisymmetric mappings (cf. [14, Lemma C]).

1.2. Extension of quasimöbius mappings. Let $(X, d)$ be a metric space. Its one-point extension is defined via

$$
\widehat{X}= \begin{cases}X, & \text { if } X \text { is bounded, } \\ X \cup\{\infty\}, & \text { if } X \text { is unbounded. }\end{cases}
$$

Let $a, b, c, d$ be points in $\widehat{X}$ with $a \neq b$ and $c \neq d$. Their cross ratio $r_{d}(a, b, c, d)$ is defined by the formula

$$
r_{d}(a, b, c, d)=\frac{d(a, c) d(b, d)}{d(a, b) d(c, d)} .
$$

If $a=c$ or $b=d$, we set $r_{d}=0$. If some of these points is $\infty$, then we omit the factors containing $\infty$. For example,

$$
r_{d}(a, b, c, \infty)=\frac{d(a, c)}{d(a, b)} .
$$

Definition 1.2. Suppose that $(X, d)$ and $\left(Y, d_{1}\right)$ are metric spaces and $\theta:[0, \infty)$ $\rightarrow[0, \infty)$ is a homeomorphism with $\theta(0)=0$. A homeomorphism $f:(\widehat{X}, d) \rightarrow\left(\widehat{Y}, d_{1}\right)$ is called $\theta$-quasimöbius, briefly $\theta$-QM, if $r_{d}(a, b, c, d) \leq t$ implies

$$
r_{d_{1}}\left(a^{\prime}, b^{\prime}, c^{\prime}, d^{\prime}\right) \leq \theta(t)
$$

for all points $a, b, c, d$ in $\widehat{X}$ and any number $t \geq 0$.

QM mappings were introduced by Väisälä in 1985 [11]. We know that every QS mapping is QM [11, Theorem 3.2] and every QM mapping between two bounded metric spaces is QS [11, p. 222]. Moreover, if a QM mapping fixes $\infty$, then it is QS [11, Theorem 3.10]. The reader is referred to [11, 12, 13] etc for more properties concerning these two classes of mappings. Also, it has been well known that the class of QM mappings has played an important role in the study of QC mappings (which is the abbreviation of quasiconformal mappings), QS mappings and their relationships (cf. $[2,9,11,14]$ etc.).

The main aim of this paper is to study the extension property of QM mappings. Our result is an analogue of Theorem B for QM mappings, which is as follows.

Theorem 1.1. Suppose that $(X, d)$ and $\left(Y, d_{1}\right)$ are metric spaces and the following conditions are satisfied:

(1) $X=X_{1} \cup X_{2}$ and $Y=Y_{1} \cup Y_{2}$ with $\angle\left(X_{1}, X_{2}\right)>0$ and $\angle\left(Y_{1}, Y_{2}\right)>0$;

(2) $X_{1} \cap X_{2}$ is $\tau$-uniformly perfect with $\tau \in(0,1)$;

(3) There is a constant $q \in(0,1]$ such that

$$
\operatorname{diam}\left(X_{1} \cap X_{2}\right) \geq q \min \left\{\operatorname{diam}\left(X_{1}\right), \operatorname{diam}\left(X_{2}\right)\right\}
$$


and

$$
\operatorname{diam}\left(Y_{1} \cap Y_{2}\right) \geq q \min \left\{\operatorname{diam}\left(Y_{1}\right), \operatorname{diam}\left(Y_{2}\right)\right\}
$$

(4) $f: X \rightarrow Y$ is a homeomorphism such that for each $i \in\{1,2\}, f\left(X_{i}\right)=Y_{i}$.

Then the restrictions $\left.f\right|_{X_{i}}(i \in\{1,2\})$ are $\theta$-quasimöbius if and only if $f$ is $\theta_{1}$ quasimöbius, where $\theta$ and $\theta_{1}$ depend on each other, and $\tau, q$, together with the angles $\angle\left(X_{1}, X_{2}\right)$ and $\angle\left(Y_{1}, Y_{2}\right)$.

Remark 1.1. By [10, Theorem 2.5], we see that the diameter condition $\operatorname{diam}\left(X_{1}\right.$ $\left.\cap X_{2}\right) \geq q \operatorname{diam}\left(X_{1}\right)$ in Theorem B guarantees the one $\operatorname{diam}\left(Y_{1} \cap Y_{2}\right) \geq q_{1} \operatorname{diam}\left(Y_{1}\right)$, where $q_{1}=\frac{1}{2 \eta(1 / q)}$, because the corresponding mappings are QS. But for QM mappings, this property is no longer valid. This can be seen from Example 4.1 below. Also, we construct three more examples to show that each of the assumptions $(1) \sim$ (3) in Theorem 1.1 cannot be removed. See Examples $4.2 \sim 4.4$ below.

We shall prove Theorem 1.1 by applying the inversions introduced by Buckley et al. in [6] or [5]. The proof will be given in Section 3. Some necessary terminologies will be introduced in Section 2, and in Section 4, four examples will be constructed.

\section{Preliminaries}

2.1. Inversions. Let $(X, d)$ denote a metric space, and let $p \in X$ be a base point. For $x, y \in X_{p}=X \backslash\{p\}$, let

and

$$
i_{p}(x, y)=\frac{d(x, y)}{d(x, p) d(y, p)}
$$

$$
d_{p}(x, y)=\inf \left\{\sum_{i=1}^{k} i_{p}\left(x_{i}, x_{i-1}\right): x=x_{0}, x_{1}, \ldots, x_{k-1}, x_{k}=y \in X_{p}\right\} .
$$

When $X$ is unbounded, for $x \in X_{p}$, we define

$$
i_{p}(x, \infty)=\frac{1}{d(x, p)} .
$$

Then we see that the definition of $d_{p}(x, y)$ using auxiliary points in $X_{p}$ is the same as the one using points in $\widehat{X}_{p}=\widehat{X} \backslash\{p\}$, and so, the distance function $d_{p}$ on $X_{p}$ extends to $\widehat{X}_{p}$.

We call

$$
\left.\operatorname{Inv}_{p}(X), d_{p}\right)=\left(\widehat{X}_{p}, d_{p}\right)
$$

the inversion of $(X, d)$ with respect to the base point $p$. In the following, sometimes, we only use $\operatorname{Inv}_{p}(X)$ to replace $\left(\operatorname{Inv}_{p}(X), d_{p}\right)$.

Let us recall the following useful properties concerning the inversions.

Theorem C. [6, Lemma 3.2] Let $(X, d)$ denote a metric space, and let $p \in X$ be a base point.

(1) For all points $x, y \in \operatorname{Inv}_{p}(X)$,

$$
\frac{1}{4} i_{p}(x, y) \leq d_{p}(x, y) \leq i_{p}(x, y)
$$

In particular, $d_{p}$ is a distance function on $\operatorname{Inv}_{p}(X)$;

(2) The identity mapping id: $\left(X_{p}, d\right) \rightarrow\left(X_{p}, d_{p}\right)$ is $\theta_{0}$-QM, where $\theta_{0}(t)=16 t$;

(3) $\left.\operatorname{Inv}_{p}(X), d_{p}\right)$ is bounded if and only if $p$ is an isolated point in $(X, d)$. 


\subsection{Uniform perfectness and density.}

Definition 2.1. A metric space $(X, d)$ is called $\tau$-uniformly perfect if there is a constant $\tau \in(0,1)$ such that for every $x \in X$ and every $r>0, \mathbf{B}(x, r) \backslash \mathbf{B}(x, \tau r) \neq \emptyset$ provided that $X \backslash \mathbf{B}(x, r) \neq \emptyset$, where $\mathbf{B}(x, r)$ denotes the metric ball $\mathbf{B}(x, r)=\{z \in$ $X: d(z, x)<r\}$.

We remark that the $\tau$-uniform perfectness implies the $\tau_{1}$-uniform perfectness when $0<\tau_{1} \leq \tau<1$.

Definition 2.2. Suppose that $(X, d)$ is a metric space, $a$ and $b \in X$, and $\left\{x_{i}\right\}_{i \in \mathbf{Z}}$ is a sequence of points in $X$ with $a \neq x_{i} \neq b$, where $\mathbf{Z}$ denotes the usual integer set.

(1) $\left\{x_{i}\right\}_{i \in \mathbf{Z}}$ is called $a$ chain joining $a$ and $b$ if $x_{i} \rightarrow a$ as $i \rightarrow-\infty$ and $x_{i} \rightarrow b$ as $i \rightarrow+\infty$. Further, if there is a constant $\sigma>1$ such that for all $i$,

$$
\left|\log r_{d}\left(a, x_{i}, x_{i+1}, b\right)\right| \leq \log \sigma,
$$

then $\left\{x_{i}\right\}_{i \in \mathbf{Z}}$ is called $a \sigma$-chain.

(2) $(X, d)$ is said to be $\sigma$-dense with $\sigma>1$ if every pair of points in $X$ can be joined by a $\sigma$-chain.

We remark that (1) every $\sigma$-dense space does not have any isolated points, and (2) each $\sigma$-dense space is $\sigma_{1}$-dense if $\sigma_{1} \geq \sigma$.

(In the rest of this paper, we make the following notational convention: Suppose $A$ denotes a condition with data $v$ and $B$ another condition with data $v_{1}$. We say that $A$ implies $B$ quantitatively if $A$ implies $B$ so that $v_{1}$ depends only on $v$. If $A$ and $B$ imply each other quantitatively, then we say that they are quantitatively equivalent.)

The next result means that the $\sigma$-density is invariant under QM mappings.

Lemma 2.1. Let $f:(X, d) \rightarrow\left(Y, d_{1}\right)$ be $\theta$-QM between two metric spaces. Then $(X, d)$ is $\sigma$-dense if and only if $\left(Y, d_{1}\right)$ is $\sigma_{1}$-dense, quantitatively.

Proof. Assume that $f$ is $\theta$-QM. Then $f^{-1}$ is $\theta_{1}$-QM with $\theta_{1}(t)=1 / \theta^{-1}(1 / t)$ (see $[11$, p. 219]). This fact implies that, to prove this lemma, it suffices to show the necessity. For this, we only need to show that for any $u^{\prime}, v^{\prime} \in Y$, there exists a $\theta(\sigma)$-chain joining them with $\theta(\sigma)>1$.

It follows from the density of $(X, d)$ that there exists a $\sigma$-chain $\left\{x_{i}\right\}_{i \in \mathbf{Z}}$ joining $u$ and $v$. Since

$$
\left|\log r_{d}\left(u, x_{i}, x_{i+1}, v\right)\right| \leq \log \sigma
$$

we see that

$$
\left|\log r_{d_{1}}\left(u^{\prime}, x_{i}^{\prime}, x_{i+1}^{\prime}, v^{\prime}\right)\right| \leq \log \theta(\sigma),
$$

and thus, $\left\{x_{i}^{\prime}\right\}_{i \in \mathbf{Z}}$ is a $\theta(\sigma)$-chain joining $u^{\prime}$ and $v^{\prime}$. Hence the proof of the lemma is complete.

Theorem D. [14, Lemma E] Let $(X, d)$ be a metric space. Then the following are quantitatively equivalent:

(1) $X$ is uniformly $\tau$-perfect;

(2) $X$ is $\sigma$-dense.

The following corollary is a direct consequence of Lemma 2.1 and Theorem D.

Corollary 2.2. Let $f:(X, d) \rightarrow\left(Y, d_{1}\right)$ be $\theta-Q M$ between two metric spaces. Then $(X, d)$ is uniformly $\tau$-perfect if and only if $\left(Y, d_{1}\right)$ is uniformly $\tau_{1}$-perfect, quantitatively. 
The following result concerns the removable property of the uniform perfectness, which is useful for the discussions in Section 3.

Lemma 2.3. Suppose that $(X, d)$ is a $\tau$-uniformly perfect metric space and $S$ denotes a finite sequence of points in $X$. Then $X \backslash S$ is $\tau^{\prime}$-uniformly perfect, where $\tau^{\prime}=\frac{\tau}{2}$.

Proof. Assume that $S=\left\{a_{1}, \ldots a_{k}\right\}$. To prove this lemma, it suffices to show the following assertion.

Assertion. For any $1 \leq i \leq k, X \backslash\left\{a_{1}, \ldots, a_{i}\right\}$ is $\mu_{i}$-uniformly perfect, where $\mu_{i}=\left(1-\frac{2^{i}-1}{2^{i+1}}\right) \tau$.

We start the proof of the assertion with two claims.

Claim 2.1. $X \backslash\left\{a_{1}\right\}$ is $\mu_{1}$-uniformly perfect, where $\mu_{1}=\left(1-\frac{1}{4}\right) \tau$.

Assume that $x \in X \backslash\left\{a_{1}\right\}, r>0$ and $\left(X \backslash\left\{a_{1}\right\}\right) \backslash \mathbf{B}(x, r) \neq \emptyset$. Then it follows from the uniform perfectness of $X$ that there is $w_{1}$ such that

$$
w_{1} \in \mathbf{B}(x, r) \backslash \mathbf{B}(x, \tau r) \text {. }
$$

If $w_{1} \neq a_{1}$, then $w_{1} \in\left(X \backslash\left\{a_{1}\right\}\right) \cap(\mathbf{B}(x, r) \backslash \mathbf{B}(x, \tau r)) \subset\left(X \backslash\left\{a_{1}\right\}\right) \cap(\mathbf{B}(x, r) \backslash$ $\left.\mathbf{B}\left(x,\left(1-\frac{1}{4}\right) \tau r\right)\right)$. Otherwise, since $X$ is uniformly perfect, we see that $a_{1}$ is not an isolated point of $X$. This implies that there is a sequence $\left\{w_{1, j}\right\}_{j=1}^{\infty} \subset X \backslash\left\{a_{1}\right\}$ such that

$$
w_{1, j} \rightarrow a_{1} \text { as } j \rightarrow \infty
$$

Let

$$
\varepsilon_{1}=\frac{1}{2} \min \left\{d\left(a_{1}, x\right)-\left(1-\frac{1}{4}\right) \tau r, r-d\left(a_{1}, x\right)\right\} .
$$

Then $\varepsilon_{1}>0$. Also, we know that there is a sufficiently large $N_{1}$ such that

$$
\left(1-\frac{1}{4}\right) \tau r<d\left(a_{1}, x\right)-\varepsilon_{1} \leq d\left(w_{1, N_{1}}, x\right) \leq d\left(a_{1}, x\right)+\varepsilon_{1}<r,
$$

from which we get

$$
w_{1, N_{1}} \in\left(X \backslash\left\{a_{1}\right\}\right) \cap\left(\mathbf{B}(x, r) \backslash \mathbf{B}\left(x,\left(1-\frac{1}{4}\right) \tau r\right)\right) .
$$

Hence the claim is proved.

Claim 2.2. $X \backslash\left\{a_{1}, a_{2}\right\}$ is $\mu_{2}$-uniformly perfect, where $\mu_{2}=\left(1-\frac{3}{8}\right) \tau$.

Let $x \in X \backslash\left\{a_{1}, a_{2}\right\}$ and $r>0$. Assume that $\left(X \backslash\left\{a_{1}, a_{2}\right\}\right) \backslash \mathbf{B}(x, r) \neq \emptyset$. It follows from Claim 2.1 that there exists a point $w_{2}$ such that

$$
w_{2} \in\left(X \backslash\left\{a_{1}\right\}\right) \cap\left(\mathbf{B}(x, r) \backslash \mathbf{B}\left(x,\left(1-\frac{1}{4}\right) \tau r\right)\right) .
$$

If $w_{2} \neq a_{2}$, then $w_{2} \in\left(X \backslash\left\{a_{1}, a_{2}\right\}\right) \cap\left(\mathbf{B}(x, r) \backslash \mathbf{B}\left(x,\left(1-\frac{1}{4}\right) \tau r\right)\right) \subset\left(X \backslash\left\{a_{1}, a_{2}\right\}\right) \cap$ $\left(\mathbf{B}(x, r) \backslash \mathbf{B}\left(x,\left(1-\frac{3}{8}\right) \tau r\right)\right)$. Otherwise, there must exist a sequence $\left\{w_{2, j}\right\}_{j=1}^{\infty} \subset$ $X \backslash\left\{a_{1}, a_{2}\right\}$ such that

Let

$$
w_{2, j} \rightarrow a_{2} \text { as } j \rightarrow \infty
$$

$$
\varepsilon_{2}=\frac{1}{2} \min \left\{d\left(a_{2}, x\right)-\left(1-\frac{3}{8}\right) \tau r, r-d\left(a_{2}, x\right)\right\} .
$$

Then $\varepsilon_{2}>0$, and we also see that there is an integer $N_{2}$ such that

$$
\left(1-\frac{3}{8}\right) \tau r<d\left(a_{2}, x\right)-\varepsilon_{2} \leq d\left(w_{2, N_{2}}, x\right) \leq d\left(a_{2}, x\right)+\varepsilon_{2}<r .
$$

This implies that

$$
w_{2, N_{2}} \in\left(X \backslash\left\{a_{1}, a_{2}\right\}\right) \cap\left(\mathbf{B}(x, r) \backslash \mathbf{B}\left(x,\left(1-\frac{3}{8}\right) \tau r\right)\right),
$$

from which the claim follows. 
By repeating the discussions as in Claims 2.1 and 2.2, we see that the assertion is true, and hence, the proof of the lemma is complete.

Remark 2.1. Let $\mathbf{Q}$ be the set of all rational numbers in $\mathbf{R}$ (the real field), and let $X=\mathrm{Q} \cup\{\sqrt{2}, \sqrt{3}\}$. Then we see that $X$ is $\tau$-uniformly perfect for every $\tau \in(0,1)$, but $X \backslash \mathbf{Q}=\{\sqrt{2}, \sqrt{3}\}$ is not $\mu$-uniformly perfect for any $\mu \in(0,1)$. This fact shows that Lemma 2.3 is invalid for the case when the removed set is infinite.

Lemma 2.4. Suppose that $(X, d)$ is a $\tau$-uniformly perfect metric space and $\operatorname{diam}(X) \geq r$, where $r$ is a positive constant. Then for every $a \in X$, there exists a point $w \in X$ such that

$$
\frac{\tau r}{4} \leq d(w, a) \leq \frac{r}{4}
$$

Proof. Since $\operatorname{diam}(X) \geq r$, for every $a \in X$, there must exist a point $w_{0} \in X$ such that

$$
d\left(w_{0}, a\right) \geq \frac{r}{3}
$$

which guarantees that $X \backslash \mathbf{B}\left(a, \frac{r}{4}\right) \neq \emptyset$. By the uniform perfectness of $X$, we know that there exists a point $w$ such that

$$
w \in \mathbf{B}\left(a, \frac{r}{4}\right) \backslash \mathbf{B}\left(a, \frac{\tau r}{4}\right) .
$$

Hence the proof of the lemma is complete.

2.3. Angles at seams, weak quasiconvexity and quasiconvexity. Suppose $(X, d)$ is a metric space. Let $X_{1}$ and $X_{2}$ be two closed subsets of $X$ with $X_{1} \cap X_{2} \neq \emptyset$. The seam is by definition the intersection $X_{1} \cap X_{2}$. Following Agard and Gehring [1], the angle $\angle\left(X_{1}, X_{2}\right)$ between $X_{1}$ and $X_{2}$ at the seam $X_{1} \cap X_{2}$ is by definition the supremum over all constants $c>0$ such that for any $\left(x_{1}, x_{2}\right) \in X_{1} \times X_{2}$,

$$
d\left(x_{1}, x_{2}\right) \geq c \inf _{y \in X_{1} \cap X_{2}}\left\{d\left(x_{1}, y\right)+d\left(x_{2}, y\right)\right\} .
$$

Definition 2.3. Suppose that $(X, d)$ is a metric space, and $X_{1}$ and $X_{2}$ are closed subsets of $X$ with $X=X_{1} \cup X_{2}$ and $X_{1} \cap X_{2} \neq \emptyset$. Let $\mu>0$. $X$ is called weakly $\mu$-quasiconvex relative to $\left(X_{1}, X_{2}\right)$ if for each pair of points $\left(x_{1}, x_{2}\right) \in\left(X_{1}, X_{2}\right)$, there exists a point $z \in X_{1} \cap X_{2}$ such that

$$
\min \left\{d\left(x_{1}, z\right), d\left(x_{2}, z\right)\right\} \leq \mu d\left(x_{1}, x_{2}\right) .
$$

Definition 2.4. A metric space $(X, d)$ is called $\nu$-quasiconvex if for any $x_{1}$ and $x_{2} \in X$, there exists a rectifiable curve $\gamma$ joining those two points such that

$$
\ell(\gamma) \leq \nu d\left(x_{1}, x_{2}\right)
$$

where $\ell(\gamma)$ means the arclength of $\gamma$.

The following lemma shows that quasiconvexity implies weak quasiconvexity.

Lemma 2.5. Suppose that $(X, d)$ is $\nu$-quasiconvex with $X=X_{1} \cup X_{2}$ and $\bar{X}_{1} \cap \bar{X}_{2} \neq \emptyset$, where the closures are taken in $X$. Then $X$ is weakly $\nu$-quasiconvex relative to $\left(\bar{X}_{1}, \bar{X}_{2}\right)$.

Proof. Let $x_{1} \in X_{1}$ and $x_{2} \in X_{2}$. Then it follows from the assumption of the quasiconvexity of $X$ that there is a curve $\gamma \subset X$ such that

$$
\ell(\gamma) \leq \nu d\left(x_{1}, x_{2}\right)
$$


The lemma easily follows since $\min \left\{d\left(z, x_{1}\right), d\left(z, x_{2}\right)\right\} \leq \ell(\gamma)$ for any $z \in \gamma \cap \bar{X}_{1} \cap$ $\bar{X}_{2}$.

Our next lemma demonstrates the equivalence between the positive angle and the weak quasiconvexity.

Lemma 2.6. Suppose that $(X, d)$ is a metric space, and $X_{1}$ and $X_{2}$ are closed subsets of $X$ with $X=X_{1} \cup X_{2}$ and $X_{1} \cap X_{2} \neq \emptyset$. Then the following statements are quantitatively equivalent.

(1) $\angle\left(X_{1}, X_{2}\right)>0$;

(2) $X$ is weakly $\mu$-quasiconvex relative to $\left(X_{1}, X_{2}\right)$.

Proof. For the proof of the necessity, let $\angle\left(X_{1}, X_{2}\right)=c$. Then $c>0$, and the proof follows from the assertion: For any $x_{1} \in X_{1}$ and $x_{2} \in X_{2}$, there exists a point $u_{1} \in X_{1} \cap X_{2}$ such that

$$
\min \left\{d\left(u_{1}, x_{1}\right), d\left(u_{1}, x_{2}\right)\right\} \leq \frac{c+1}{2 c} d\left(x_{1}, x_{2}\right) .
$$

To prove this assertion, we only need to consider the case $x_{1} \neq x_{2}$. Since the assumption $c>0$ implies that

$$
c \inf _{y \in X_{1} \cap X_{2}}\left\{d\left(y, x_{1}\right)+d\left(y, x_{2}\right)\right\} \leq d\left(x_{1}, x_{2}\right),
$$

and because there exists a point $y_{1} \in X_{1} \cap X_{2}$ such that

$$
d\left(y_{1}, x_{1}\right)+d\left(y_{1}, x_{2}\right) \leq \inf _{y \in X_{1} \cap X_{2}}\left\{d\left(y, x_{1}\right)+d\left(y, x_{2}\right)\right\}+d\left(x_{1}, x_{2}\right),
$$

we know that

$$
\min \left\{d\left(y_{1}, x_{1}\right), d\left(y_{1}, x_{2}\right)\right\} \leq \frac{d\left(y_{1}, x_{1}\right)+d\left(y_{1}, x_{2}\right)}{2} \leq \frac{c+1}{2 c} d\left(x_{1}, x_{2}\right) .
$$

By letting $u_{1}=y_{1}$, we see that (2.1) is true.

To prove the sufficiency, we only need to show that for any $x_{1} \in X_{1}$ and $x_{2} \in X_{2}$,

$$
\frac{1}{2(\mu+1)} \inf _{y \in X_{1} \cap X_{2}}\left\{d\left(y, x_{1}\right)+d\left(y, x_{2}\right)\right\} \leq d\left(x_{1}, x_{2}\right) .
$$

Since $X$ is weakly $\mu$-quasiconvex relative to $\left(X_{1}, X_{2}\right)$, there exists a point $y_{0} \in$ $X_{1} \cap X_{2}$ such that

$$
\max \left\{d\left(y_{0}, x_{1}\right), d\left(y_{0}, x_{2}\right)\right\} \leq(\mu+1) d\left(x_{1}, x_{2}\right) .
$$

Obviously, (2.2) follows from the following inequality:

$$
\inf _{y \in X_{1} \cap X_{2}}\left\{d\left(y, x_{1}\right)+d\left(y, x_{2}\right)\right\} \leq 2 \max \left\{d\left(y_{0}, x_{1}\right), d\left(y_{0}, x_{2}\right)\right\} .
$$

Thus the lemma is proved.

\section{Quasimöbius mappings and unions}

Lemma 3.1. Suppose $X_{1}$ and $X_{2}$ are closed subsets of $X$ with $X=X_{1} \cup X_{2}$ and $X_{1} \cap X_{2} \neq \emptyset$, where $(X, d)$ is a metric space. If $\operatorname{diam}\left(X_{1} \cap X_{2}\right) \geq q \operatorname{diam}\left(X_{1}\right)$ and $X_{1} \cap X_{2}$ is $\tau$-uniformly perfect with $q \in(0,1]$ and $\tau \in(0,1)$, then for any $x_{11}$, $x_{12} \in X_{1}$ and $\xi \in X_{1} \cap X_{2}$, there exists a point $\zeta \in X_{1} \cap X_{2}$ such that

$$
\frac{1}{L} d\left(x_{11}, x_{12}\right) \leq d(\xi, \zeta) \leq L d\left(x_{11}, x_{12}\right),
$$

where $L=\max \left\{\frac{4}{q}, \frac{1}{\tau}\right\}$. 
Proof. If $\operatorname{diam}\left(X_{1} \cap X_{2}\right)<\frac{1}{\tau} d\left(x_{11}, x_{12}\right)$, since there is a point $\zeta \in X_{1} \cap X_{2}$ such that

$$
\frac{1}{4} \operatorname{diam}\left(X_{1} \cap X_{2}\right) \leq d(\zeta, \xi) \leq \operatorname{diam}\left(X_{1} \cap X_{2}\right)
$$

it follows from the assumption $\operatorname{diam}\left(X_{1} \cap X_{2}\right) \geq q \operatorname{diam}\left(X_{1}\right)$ of the lemma that

$$
\frac{q}{4} d\left(x_{11}, x_{12}\right) \leq d(\zeta, \xi) \leq \frac{1}{\tau} d\left(x_{11}, x_{12}\right) .
$$

Now, we assume that $\operatorname{diam}\left(X_{1} \cap X_{2}\right) \geq \frac{1}{\tau} d\left(x_{11}, x_{12}\right)$. Then Lemma 2.4 guarantees that there exists $\zeta \in X_{1} \cap X_{2}$ such that

$$
\frac{1}{4} d\left(x_{11}, x_{12}\right) \leq d(\zeta, \xi) \leq \frac{1}{4 \tau} d\left(x_{11}, x_{12}\right) .
$$

Easily, the lemma follows from (3.1) and (3.2).

Lemma 3.2. Suppose that $(X, d)$ is a metric space and the following conditions are satisfied:

(1) $X_{1}$ and $X_{2}$ are closed subsets of $X$ with $X=X_{1} \cup X_{2}$ and $X_{1} \cap X_{2} \neq \emptyset$;

(2) $X$ is weakly $\mu$-quasiconvex relative to $\left(X_{1}, X_{2}\right)$ with $\mu>0$;

(3) There is a constant $q \in(0,1]$ such that

$$
\operatorname{diam}\left(X_{1} \cap X_{2}\right) \geq q \min \left\{\operatorname{diam}\left(X_{1}\right), \operatorname{diam}\left(X_{2}\right)\right\}
$$

(4) $X_{1} \cap X_{2}$ is $\tau$-uniformly perfect with $\tau \in(0,1)$.

Then for any $p \in X_{1} \cap X_{2}$, the following hold:

(i) $X_{p}$ is weakly $\mu_{1}$-quasiconvex relative to $\left(\left(X_{1}\right)_{p},\left(X_{2}\right)_{p}\right)$ with respect to the metric $d_{p}$, where $\mu_{1}=16 L(\mu+1)(L+1)$ and $L$ is the constant from Lemma 3.1;

(ii) $\operatorname{diam}_{p}\left(\left(X_{i}\right)_{p}\right)=\operatorname{diam}_{p}\left(\left(X_{1} \cap X_{2}\right)_{p}\right)$ for $i \in\{1,2\}$;

(iii) $\left(X_{1} \cap X_{2}\right)_{p}$ is $\tau_{1}$-uniformly perfect with respect to the metric $d_{p}$, where $\tau_{1}$ depends only on $\tau$.

Here $\operatorname{diam}_{p}(M)$ denotes the diameter of a set $M$ with respect to the metric $d_{p}$.

Proof. By the assumption (4) of the lemma, we see that $X_{1} \cap X_{2}$ has no isolated point. Then Theorem $\mathrm{C}(3)$ ensures that all the quantities $\operatorname{diam}_{p}\left(\left(X_{i}\right)_{p}\right)(i \in\{1,2\})$ and $\operatorname{diam}_{p}\left(\left(X_{1} \cap X_{2}\right)_{p}\right)$ are $\infty$, and so, the statement (ii) of the lemma is true.

Again, the assumption (4) of the lemma along with Lemma 2.3 guarantees that $\left(X_{1} \cap X_{2}\right)_{p}$ is $\tau^{\prime}$-uniformly perfect, where $\tau^{\prime}=\frac{\tau}{2}$. Then the statement (iii) of the lemma follows from Theorem $\mathrm{C}(2)$, Theorem D and Lemma 2.1.

To complete the proof, it remains to prove the statement (i) of the lemma. We are going to prove that for any $x_{1} \in\left(X_{1}\right)_{p}$ and $x_{2} \in\left(X_{2}\right)_{p}$, there exists $\zeta \in\left(X_{1} \cap X_{2}\right)_{p}$ such that

$$
\min \left\{d_{p}\left(x_{1}, \zeta\right), d_{p}\left(x_{2}, \zeta\right)\right\} \leq \mu_{1} d_{p}\left(x_{1}, x_{2}\right),
$$

where $\mu_{1}=16 L(\mu+1)(L+1)$.

If $x_{1} \in\left(X_{1} \cap X_{2}\right)_{p}$ (resp. $\left.x_{2} \in\left(X_{1} \cap X_{2}\right)_{p}\right)$, by taking $\zeta=x_{1}\left(\operatorname{resp} . \zeta=x_{2}\right),(3.3)$ follows.

If $x_{1} \in\left(X_{1}\right)_{p} \backslash\left(\left(X_{1}\right)_{p} \cap\left(X_{2}\right)_{p}\right)$ and $x_{2} \in\left(X_{2}\right)_{p} \backslash\left(\left(X_{1}\right)_{p} \cap\left(X_{2}\right)_{p}\right)$, it follows from the assumption (2) of the lemma that there exists $z_{0} \in X_{1} \cap X_{2}$ such that

$$
\max \left\{d\left(x_{1}, z_{0}\right), d\left(x_{2}, z_{0}\right)\right\} \leq(\mu+1) d\left(x_{1}, x_{2}\right) .
$$

Without loss of generality, we may assume that

$$
\min \left\{\operatorname{diam}\left(X_{1}\right), \operatorname{diam}\left(X_{2}\right)\right\}=\operatorname{diam}\left(X_{1}\right) .
$$

We divide the discussions into two cases: $d\left(z_{0}, p\right) \leq 3 d\left(x_{1}, z_{0}\right)$ and $d\left(z_{0}, p\right)>3 d\left(x_{1}, z_{0}\right)$. 
For the former case, we see from (3.4) that

$$
\begin{aligned}
\max \left\{d\left(x_{1}, p\right), d\left(x_{2}, p\right)\right\} & \leq \max \left\{d\left(x_{1}, z_{0}\right), d\left(x_{2}, z_{0}\right)\right\}+d\left(z_{0}, p\right) \\
& \leq 4(\mu+1) d\left(x_{1}, x_{2}\right) .
\end{aligned}
$$

By replacing $x_{11}, x_{12}$ and $\xi$ in Lemma 3.1 by $x_{1}, p, p$, respectively, it follows from Lemma 3.1 that there exists $z_{1} \in X_{1} \cap X_{2}$ such that

$$
\frac{1}{L} d\left(x_{1}, p\right) \leq d\left(z_{1}, p\right) \leq L d\left(x_{1}, p\right)
$$

and so, (3.5) gives

$$
d\left(z_{1}, p\right) \leq 4 L(\mu+1) d\left(x_{1}, x_{2}\right) .
$$

Thus the assumption of this case and (3.4) lead to

$$
d\left(x_{2}, z_{1}\right) \leq d\left(x_{2}, z_{0}\right)+d\left(z_{0}, p\right)+d\left(z_{1}, p\right) \leq 4(\mu+1)(L+1) d\left(x_{1}, x_{2}\right) .
$$

Thus we deduce from Theorem $\mathrm{C}(1)$ and (3.5) that

$$
d_{p}\left(x_{2}, z_{1}\right) \leq \frac{d\left(x_{2}, z_{1}\right)}{d\left(x_{2}, p\right) d\left(z_{1}, p\right)} \leq 16 L(\mu+1)(L+1) d_{p}\left(x_{1}, x_{2}\right) .
$$

Since $x_{1} \neq p$, again, it follows from (3.5) that $z_{1} \neq p$. By taking $\zeta=z_{1}$, (3.3) follows.

For the latter case, i.e., $d\left(z_{0}, p\right)>3 d\left(x_{1}, z_{0}\right)$, we know that $z_{0} \neq p$ and

$$
d\left(x_{1}, p\right) \leq d\left(z_{0}, p\right)+d\left(x_{1}, z_{0}\right) \leq \frac{4}{3} d\left(z_{0}, p\right) .
$$

Then Theorem $\mathrm{C}(1)$ and (3.4) lead to

$$
d_{p}\left(x_{2}, z_{0}\right) \leq \frac{d\left(x_{2}, z_{0}\right)}{d\left(x_{2}, p\right) d\left(z_{0}, p\right)} \leq \frac{16}{3}(\mu+1) d_{p}\left(x_{1}, x_{2}\right) .
$$

By taking $\zeta=z_{0}$, we know that (3.3) is true. Hence the lemma is proved.

Proof of Theorem 1.1. The sufficiency is obvious. To prove the necessity, for convenience, let $f_{i}=\left.f\right|_{X_{i}}$ for $i=1,2$. Now, we assume that both $f_{1}$ and $f_{2}$ are $\theta$-QM. Let $p \in X_{1} \cap X_{2}$, and let

$$
g=\mathrm{id}_{2} \circ f \circ \mathrm{id}_{1}^{-1}:\left(X_{p}, d_{p}\right) \rightarrow\left(Y_{p^{\prime}}, d_{1, p^{\prime}}\right),
$$

where $p^{\prime}=f(p)$, both $\mathrm{id}_{1}:\left(X_{p}, d\right) \rightarrow\left(X_{p}, d_{p}\right)$ and $\mathrm{id}_{2}:\left(Y_{p^{\prime}}, d_{1}\right) \rightarrow\left(Y_{p^{\prime}}, d_{1, p^{\prime}}\right)$ are the identity mappings. Also, we use $x$ (resp. $y$ ) to denote both $x$ and its $\operatorname{image~}_{1} \operatorname{id}_{1}(x)$ for any $x \in X_{p}$ (resp. both $y$ and its image $i d_{2}(y)$ for any $\left.y \in Y_{p^{\prime}}\right)$. Furthermore, for $i=1,2$, let

$$
g_{i}=\left.g\right|_{\left(X_{i}\right)_{p}} .
$$

Then $g_{i}(x)=x^{\prime}$ for all $x \in\left(X_{i}\right)_{p}$, where $x^{\prime}=f(x)$.

To finish the proof, we need the following claim.

Claim 3.1. (a) $g_{i}$ is $\theta_{1}$-QS, where $\theta_{1}(t)=16 \theta(16 t)$;

(b) $g$ is $\eta_{2}-Q S$, where $\eta_{2}$ depends on $\theta, \tau$, $q$, together with the angles $\angle\left(X_{1}, X_{2}\right)$ and $\angle\left(Y_{1}, Y_{2}\right)$.

First, we prove (a). We see from Theorem $\mathrm{C}(2)$ that for each $i \in\{1,2\}, g_{i}$ is $\theta_{1}$-QM. Without loss generality, we may assume that $i=1$. To prove this statement, we only need to show that for every triple $\{x, a, b\}$ in $\left(X_{1}\right)_{p}$,

$$
\frac{d_{1, p^{\prime}}\left(g_{1}(x), g_{1}(a)\right)}{d_{1, p^{\prime}}\left(g_{1}(x), g_{1}(b)\right)} \leq 16 \theta\left(16 \frac{d_{p}(x, a)}{d_{p}(x, b)}\right) \text {. }
$$


By the uniform perfectness of $X_{1} \cap X_{2}$, we know that there is a sequence $\left\{p_{n}\right\} \subset$ $\left(X_{1} \cap X_{2}\right)_{p}$ such that $d\left(p_{n}, p\right) \rightarrow 0$ as $n \rightarrow \infty$. Obviously, $\left\{p_{n}^{\prime}\right\} \subset\left(Y_{1} \cap Y_{2}\right)_{p^{\prime}}$ and $d_{1}\left(p_{n}^{\prime}, p^{\prime}\right) \rightarrow 0$ as $n \rightarrow \infty$, where $p_{n}^{\prime}=f\left(p_{n}\right)$. Since $g_{1}$ is $\theta_{1}$-QM, we have

$$
\frac{d_{1, p^{\prime}}\left(g_{1}(x), g_{1}(a)\right) d_{1, p^{\prime}}\left(g_{1}(b), g_{1}\left(p_{n}\right)\right)}{d_{1, p^{\prime}}\left(g_{1}(x), g_{1}(b)\right) d_{1, p^{\prime}}\left(g_{1}(x), g_{1}\left(p_{n}\right)\right)} \leq 16 \theta\left(16 \frac{d_{p}(x, a) d_{p}\left(b, p_{n}\right)}{d_{p}(x, b) d_{p}\left(x, p_{n}\right)}\right) .
$$

Moreover, it follows from Theorem $\mathrm{C}(1)$ that all the quantities

$$
d_{p}\left(x, p_{n}\right), \quad d_{p}\left(b, p_{n}\right), \quad d_{1, p^{\prime}}\left(g_{1}(x), g_{1}\left(p_{n}\right)\right) \text { and } d_{1, p^{\prime}}\left(g_{1}(b), g_{1}\left(p_{n}\right)\right)
$$

tend to $\infty$ as $n \rightarrow \infty$. Thus (3.8) follows from (3.9) by letting $n$ tend to $\infty$.

Next, we shall apply Theorem B to prove (b). For this, we need to check that all the assumptions in Theorem B are satisfied. Since Corollary 2.2 guarantees that $Y_{1} \cap Y_{2}$ is $\tau_{2}$-uniformly perfect, where $\tau_{2}$ depends only on $\theta$ and $\tau$, we see from Lemmas 2.6 and 3.2, together with the statement (a) of the claim, that all the assumptions in Theorem B are satisfied. By Theorem B, we know that the statement (b) of the claim is true, and thus, the claim is proved.

We are ready to finish the proof of the theorem. Since Claim 3.1(b) implies that $\left.f\right|_{X_{p}}=\mathrm{id}_{2}^{-1} \circ g \circ \mathrm{id}_{1}$ is $\theta_{2}$-QM, where $\theta_{2}$ depends only on $\eta_{2}$, and since $p$ is not an isolated point of $X$, we see from the homeomorphism of $f$ that $f$ is also $\theta_{2}$-QM. Now, the proof of the theorem is complete.

\section{Some examples}

Throughout this section, $\mathbf{C}$ denotes the complex plane and $z=x+i y$ stands for a point in $\mathbf{C}$, where $x$ and $y \in \mathbf{R}, O$ denotes the coordinate origin in $\mathbf{C}$, and the metric $d=|\cdot|$ is the usual Euclidean metric.

In this section, our purpose is to construct four examples. The first example shows that the diameter condition in Theorem B is not invariant with respect to QM mappings. The remaining three examples demonstrate that each of the first three assumptions in Theorem 1.1 cannot be removed.

Example 4.1. Let

$$
X=X_{1} \cup X_{2},
$$

where $X_{1}=\left(I_{1} \cup I_{2}\right) \backslash\{O\}, X_{2}=\left(I_{3} \cup I_{4}\right) \backslash\{O\}$,

$$
\begin{gathered}
I_{1}=\left\{z \in \mathbf{C}: x^{2}+y^{2} \leq 4, x \geq 0, y \geq 0\right\}, \\
I_{2}=\left\{z \in \mathbf{C}: 1 \leq x^{2}+y^{2} \leq 4, x \leq 0, y \leq 0\right\}, \\
I_{3}=\left\{z \in \mathbf{C}: x^{2}+y^{2} \leq 4, x \leq 0, y \leq 0\right\}
\end{gathered}
$$

and

$$
I_{4}=\left\{z \in \mathbf{C}: 1 \leq x^{2}+y^{2} \leq 4, x \geq 0, y \geq 0\right\}
$$

(see Figure 1), and let

$$
f: X \rightarrow Y
$$

with $f(z)=\frac{z}{|z|^{2}}$ and $Y=f(X)$. Then we have the following conclusions.

(1) The homeomorphism $f$ is $\theta_{3}$-QM, where $\theta_{3}(t)=81 t$;

(2) $\operatorname{diam}\left(X_{1}\right)=\operatorname{diam}\left(X_{2}\right)=\operatorname{diam}\left(X_{1} \cap X_{2}\right)=4$;

(3) $\operatorname{diam}\left(f\left(X_{1}\right)\right)=\operatorname{diam}\left(f\left(X_{2}\right)\right)=\infty$, but $\operatorname{diam}\left(f\left(X_{1}\right) \cap f\left(X_{2}\right)\right)=2$.

Proof. The first assertion follows from [11, p. 220], and the rest two assertions are obvious. 


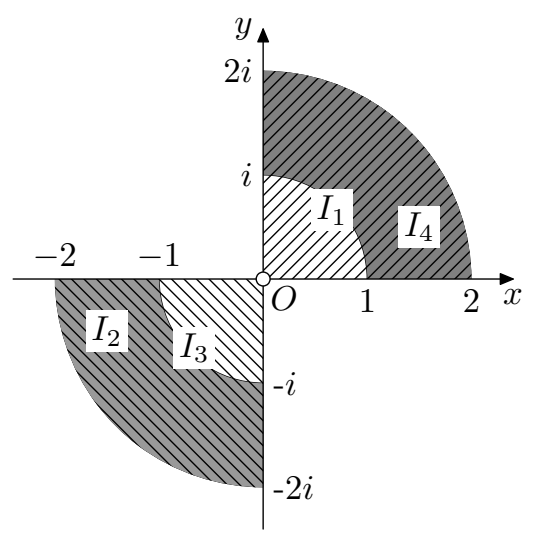

Figure 1. Example 4.1.

The following example shows that the assumption (1) in Theorem 1.1 cannot be removed.

Example 4.2. Let

$$
X=X_{1} \cup X_{2},
$$

where $X_{1}=J_{1} \cup J_{3}, X_{2}=J_{2} \cup J_{3}, J_{1}=\left\{z \in \mathbf{C}: \frac{1}{64}<x^{2}+y^{2}<1, x>0, y>0\right\}$,

$$
J_{2}=\left\{z \in \mathbf{C}: x^{2}+y^{2}<1, x>0, y<0\right\} \text { and } J_{3}=\left\{z \in \mathbf{C}: x^{2}+y^{2}=1, x>0\right\}
$$

(see Figure 2), and let

$$
f: X \rightarrow Y
$$

where $\left.f\right|_{X_{1}}(z)=\frac{z}{|z|^{2}},\left.f\right|_{X_{2}}(z)=z$ and $Y=f(X)$. Then the following statements hold.

(a) $X=X_{1} \cup X_{2}$ is not weakly $\mu$-quasiconvex relative to $\left(X_{1}, X_{2}\right)$ for any $\mu>0$;

(b) Both $X_{1} \cap X_{2}$ and $f\left(X_{1}\right) \cap f\left(X_{2}\right)$ are connected;

(c) $\operatorname{diam}\left(X_{1} \cap X_{2}\right)=\operatorname{diam}\left(X_{1}\right)=\operatorname{diam}\left(X_{2}\right)=2, \operatorname{diam}\left(f\left(X_{1}\right) \cap f\left(X_{2}\right)\right)=$ $\operatorname{diam}\left(f\left(X_{2}\right)\right)=2$ and $\operatorname{diam}\left(f\left(X_{1}\right)\right)=8 \sqrt{2}$;

(d) Both $\left.f\right|_{X_{1}}$ and $\left.f\right|_{X_{2}}$ are $\theta_{3}$-QM, where $\theta_{3}(t)=81 t$;

(e) The homeomorphism $f$ is not $\theta$-QM for any homeomorphism $\theta$.

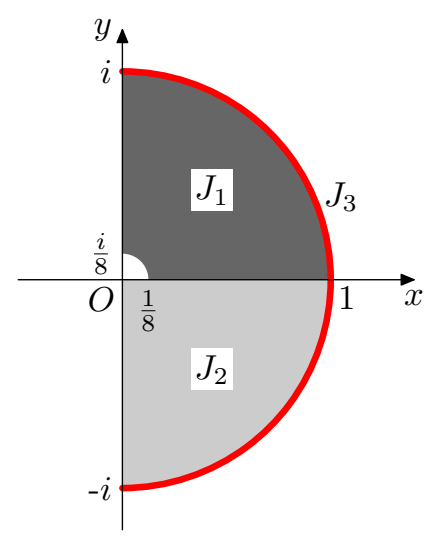

Figure 2. Example 4.2.

Proof. To prove the first statement, let $z_{1}=\frac{1}{2}+i s$ and $z_{2}=\frac{1}{2}-i s$, where $0<s<\frac{1}{2}$. Then we have

$$
\left|z_{1}-z_{2}\right|=2 s
$$


Moreover, for any $z \in X_{1} \cap X_{2}=J_{3}$, we have

$$
\min \left\{\left|z_{1}-z\right|,\left|z_{2}-z\right|\right\} \geq \frac{2-\sqrt{1+4 s^{2}}}{2}
$$

By letting $s \rightarrow 0$, we see that $X$ is not weakly $\mu$-quasiconvex relative to $\left(X_{1}, X_{2}\right)$ for any $\mu>0$.

The second and the third statements are obvious, and the forth one easily follows from [11, p. 220]. Now, we remain to show the last statement. For this, let

$$
z_{3}=\frac{1}{4}-i s, \quad z_{4}=\frac{1}{2}-i s, \quad z_{5}=\frac{1}{4}+i s \quad \text { and } \quad z_{6}=\frac{1}{2}+i s,
$$

where $0<s<\frac{1}{2}$. By elementary computations, we obtain

$$
r=\frac{\left|z_{3}-z_{5}\right|\left|z_{4}-z_{6}\right|}{\left|z_{3}-z_{4}\right|\left|z_{5}-z_{6}\right|}=64 s^{2} \text { and } r_{1}=\frac{\left|z_{3}^{\prime}-z_{5}^{\prime}\right|\left|z_{4}^{\prime}-z_{6}^{\prime}\right|}{\left|z_{3}^{\prime}-z_{4}^{\prime}\right|\left|z_{5}^{\prime}-z_{6}^{\prime}\right|}=\frac{2 \lambda_{1}(s) \lambda_{2}(s)}{\sqrt{\left(1+16 s^{2}\right)\left(1+4 s^{2}\right)}}
$$

where

$$
\lambda_{1}(s)=\sqrt{\left(\frac{15}{4}-4 s^{2}\right)^{2}+\left(17+16 s^{2}\right)^{2} s^{2}} \text { and } \lambda_{2}(s)=\sqrt{\left(\frac{3}{2}-2 s^{2}\right)^{2}+\left(5+4 s^{2}\right)^{2} s^{2}} .
$$

Since $0<s<\frac{1}{2}$, we see that

$$
r_{1} \geq \frac{9}{8} \sqrt{10}
$$

and thus, the fact $r \rightarrow 0$ as $s \rightarrow 0$ implies that $f$ is not $\theta$-QM for any homeomorphism $\theta$.

The purpose of our next example is to illustrate that the assumption (2) in Theorem 1.1 cannot be removed.

Example 4.3. Let

$$
X=X_{1} \cup X_{2}
$$

where $X_{1}=\left\{z \in \mathbf{C}: x^{2}+y^{2} \leq 1, x \leq 0\right\} \cup\left\{p_{0}\right\}, p_{0}$ denotes the point $(1,0)$ and $X_{2}=\{z \in \mathbf{C}: x \geq 0, y=0\}$ (see Figure 3), and let

$$
f: X \rightarrow X
$$

where $\left.f\right|_{X_{1}}(z)=z$ and $\left.f\right|_{X_{2}}(z)=z^{2}$. Then the following statements hold.

(I) $f\left(X_{i}\right)=X_{i}$ for each $i=1,2$;

(II) $X=X_{1} \cup X_{2}$ is weakly 1-quasiconvex relative to $\left(X_{1}, X_{2}\right)$;

(III) $X_{1} \cap X_{2}$ is not $\tau$-uniformly perfect for any $\tau \in(0,1)$;

(IV) $\operatorname{diam}\left(X_{1} \cap X_{2}\right)=q \min \left\{\operatorname{diam}\left(X_{1}\right), \operatorname{diam}\left(X_{2}\right)\right\}=1$, where $q=\frac{1}{2}$;

(V) $\left.f\right|_{X_{1}}$ and $\left.f\right|_{X_{2}}$ are $\theta_{4}$-QM, where

$$
\theta_{4}(t)=4 t^{3}+8 t^{2} \sqrt{t}+135 t^{2}+8 t \sqrt{t}+90 t+16 \sqrt{t}
$$

(VI) The homeomorphism $f$ is not $\theta$-QM for any homeomorphism $\theta$. 


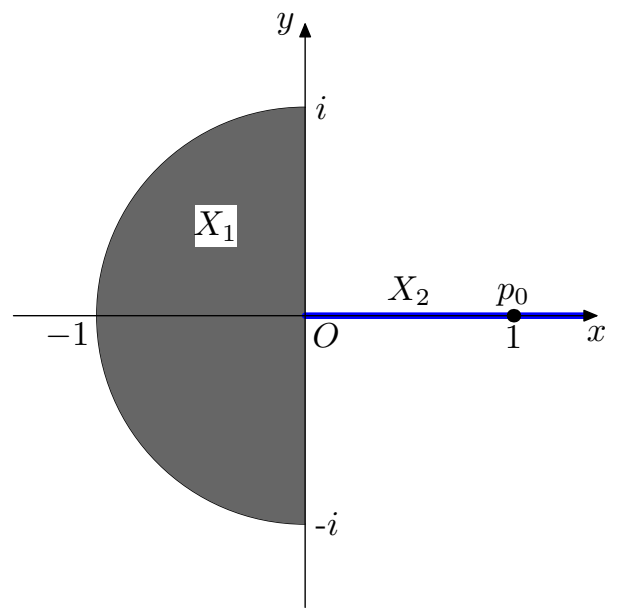

Figure 3. Example 4.3.

Proof. The first statement is obvious. To prove the second statement, it suffices to show that for any $z_{1} \in X_{1}$ and $z_{2} \in X_{2}$, there exists $z^{*} \in X_{1} \cap X_{2}\left(=\left\{O, p_{0}\right\}\right)$ such that

$$
\min \left\{\left|z_{1}-z^{*}\right|,\left|z_{2}-z^{*}\right|\right\} \leq\left|z_{1}-z_{2}\right| .
$$

If $z_{1} \in X_{1} \cap X_{2}$ (resp. $z_{2} \in X_{1} \cap X_{2}$ ), let $z^{*}=z_{1}$ (resp. $z^{*}=z_{2}$ ). Then (4.1) is obvious.

If $z_{1} \in X_{1} \backslash\left(X_{1} \cap X_{2}\right)$ and $z_{2} \in X_{2} \backslash\left(X_{1} \cap X_{2}\right)$, then we easily know that the angle formed by the vectors $\overrightarrow{O z_{1}}$ and $\overrightarrow{O z_{2}}$ is at least $\frac{\pi}{2}$. By letting $z^{*}=O$, we see that (4.1) is true.

The third and forth statements are obvious. For the fifth one, obviously, $\left.f\right|_{X_{1}}$ is $\theta_{1}$-QM, where $\theta_{5}(t)=t$. Hence it is $\theta_{4}$-QM, where

$$
\theta_{4}(t)=4 t^{3}+8 t^{2} \sqrt{t}+135 t^{2}+8 t \sqrt{t}+90 t+16 \sqrt{t} .
$$

About $\left.f\right|_{X_{2}}$, we first show that $\left.f\right|_{X_{2}}$ is $\eta$-QS, where

$$
\eta(t)=t(t+2) \text {. }
$$

Let $z_{1}=\left(x_{1}, 0\right), z_{2}=\left(x_{2}, 0\right), z_{3}=\left(x_{3}, 0\right) \in X_{2}$ with $z_{1} \neq z_{3}$. Then

$$
\frac{\left|f\left(z_{1}\right)-f\left(z_{2}\right)\right|}{\left|f\left(z_{1}\right)-f\left(z_{3}\right)\right|}=\frac{\left|x_{1}^{2}-x_{2}^{2}\right|}{\left|x_{1}^{2}-x_{3}^{2}\right|}=\frac{\left(x_{1}+x_{2}\right)\left|z_{1}-z_{2}\right|}{\left(x_{1}+x_{3}\right)\left|z_{1}-z_{3}\right|} .
$$

Since

$$
\frac{x_{1}+x_{2}}{x_{1}+x_{3}} \leq 2+\frac{\left|z_{1}-z_{2}\right|}{\left|z_{1}-z_{3}\right|}
$$

we see that $\left.f\right|_{X_{2}}$ is $\eta$-QS, where $\eta(t)=t(t+2)$. Thus the similar reasoning as in the proof of [11, Theorem 3.2] ensures that $\left.f\right|_{X_{2}}$ is $\theta_{4}$-QM.

To finish the proof, it remains to show the last statement. For this, let

$$
z_{4}=0, z_{5}=s, z_{6}=-s \text { and } z_{7}=-2 s,
$$

where $0<s<1$. By elementary computations, we obtain

$$
r=\frac{\left|z_{4}-z_{6}\right|\left|z_{5}-z_{7}\right|}{\left|z_{4}-z_{5}\right|\left|z_{6}-z_{7}\right|}=3 \text { and } r_{1}=\frac{\left|z_{4}^{\prime}-z_{6}^{\prime}\right|\left|z_{5}^{\prime}-z_{7}^{\prime}\right|}{\left|z_{4}^{\prime}-z_{5}^{\prime}\right|\left|z_{6}^{\prime}-z_{7}^{\prime}\right|}=\frac{s+2}{s} \text {. }
$$

Now, it follows from the fact $r_{1} \rightarrow+\infty$ as $s \rightarrow 0$ that $f$ is not $\theta$-QM for any homeomorphism $\theta$. Hence the proof of the example is complete. 
By the next example, we know that the assumption (3) in Theorem 1.1 cannot be removed.

Example 4.4. Let

$$
X=X_{1} \cup X_{2}
$$

where $X_{1}=\left\{z \in \mathbf{C}: x^{2}+y^{2} \geq 1, x \geq 0, y \geq 0\right\}, X_{2}=K_{1} \cup K_{2}, K_{1}=\{z \in$ C: $\left.x^{2}+y^{2}=1, x \geq 0, y \geq 0\right\}$ and $K_{2}=\{z \in \mathbf{C}: x=1, y \leq 0\}$ (see Figure 4), and let

$$
f: X \rightarrow Y
$$

where $\left.f\right|_{X_{1}}(z)=\frac{z}{|z|^{2}},\left.f\right|_{X_{2}}(z)=z$ and $Y=f(X)$. Then the following statements hold.

(i) $X=X_{1} \cup X_{2}$ is weakly 1-quasiconvex relative to $\left(X_{1}, X_{2}\right)$ and $Y=f\left(X_{1}\right) \cup$ $f\left(X_{2}\right)$ is weakly 1-quasiconvex relative to $\left(f\left(X_{1}\right), f\left(X_{2}\right)\right)$;

(ii) Both $X_{1} \cap X_{2}$ and $f\left(X_{1}\right) \cap f\left(X_{2}\right)$ are connected;

(iii) $\operatorname{diam}\left(X_{1}\right)=\operatorname{diam}\left(X_{2}\right)=\infty, \operatorname{diam}\left(X_{1} \cap X_{2}\right)=\sqrt{2}$, and $\operatorname{diam}\left(f\left(X_{1}\right) \cap\right.$ $\left.f\left(X_{2}\right)\right)=\min \left\{\operatorname{diam}\left(f\left(X_{1}\right)\right), \operatorname{diam}\left(f\left(X_{2}\right)\right)\right\}=\sqrt{2}$

(iv) Both $\left.f\right|_{X_{1}}$ and $\left.f\right|_{X_{2}}$ are $\theta_{3}$-QM, where $\theta_{3}(t)=81 t$;

(v) $f$ is not $\theta$-QM for any homeomorphism $\theta$.

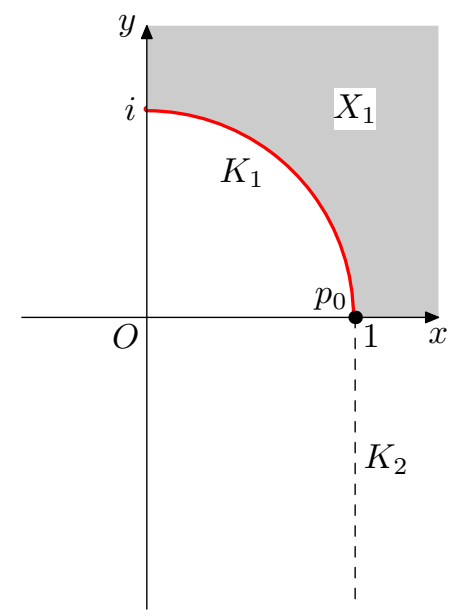

Figure 4. Example 4.4.

Proof. To prove the first statement, we only need to illustrate the relatively weak quasiconvexity of $X$ since the proof of that of $Y$ is similar. To reach this goal, it suffices to show that for any $z_{1} \in X_{1}$ and $z_{2} \in X_{2}$, there exists $z_{0} \in X_{1} \cap X_{2}\left(=K_{1}\right)$ such that

$$
\min \left\{\left|z_{1}-z_{0}\right|,\left|z_{2}-z_{0}\right|\right\} \leq\left|z_{1}-z_{2}\right| .
$$

If $z_{1} \in K_{1}$ (resp. $z_{2} \in K_{1}$ ), let $z_{0}=z_{1}$ (resp. $z_{0}=z_{2}$ ). Then (4.2) is obvious.

If $z_{1} \in X_{1} \backslash K_{1}$ and $z_{2} \in X_{2} \backslash K_{1}$, then we easily know that the angle formed by the vectors $\overrightarrow{p_{0} z_{1}}$ and $\overrightarrow{p_{0} z_{2}}$ is at least $\frac{\pi}{2}$ ( we recall that $p_{0}$ denotes the point $(1,0)$ in C). This fact guarantees that (4.2) holds by letting $z_{0}=p_{0}$.

The second and third statements are obvious, and the forth one follows from [11, p. 220]. To finish the proof, it remains to show the last statement. For this, let

$$
z_{3}=\frac{1+i}{2} t, \quad z_{4}=(1+i) t, \quad z_{5}=1-10 i \text { and } z_{6}=1-t i
$$


where $t>10$ is an integer. By elementary computations, we obtain

$$
r=\frac{\left|z_{3}-z_{5}\right|\left|z_{4}-z_{6}\right|}{\left|z_{3}-z_{4}\right|\left|z_{5}-z_{6}\right|}=\frac{\sqrt{(t-2)^{2}+(t+20)^{2}} \sqrt{(t-1)^{2}+4 t^{2}}}{\sqrt{2} t(t-10)}
$$

and

$$
r_{1}=\frac{\left|z_{3}^{\prime}-z_{5}^{\prime}\right|\left|z_{4}^{\prime}-z_{6}^{\prime}\right|}{\left|z_{3}^{\prime}-z_{4}^{\prime}\right|\left|z_{5}^{\prime}-z_{6}^{\prime}\right|}=\frac{\sqrt{(t-1)^{2}+(10 t+1)^{2}} \sqrt{(2 t-1)^{2}+\left(2 t^{2}+1\right)^{2}}}{\sqrt{2} t(t-10)} .
$$

Now, it follows from the fact $r \rightarrow \sqrt{5}$ and $r_{1} \rightarrow+\infty$ as $t \rightarrow+\infty$ that $f$ is not $\theta$-QM for any homeomorphism $\theta$. Hence the proof of the example is complete.

Funding. The research was partly supported by NSFs of China (Nos. 11822105, 11571216, 11671127 and 11720101003), the excellent youth foundation of Hunan scientific committee (No. 2017JJ1019) and STU SRFT.

Acknowledgements. The authors would like to express their deep gratitude to the referee for his or her careful reading of the paper and his useful suggestions and remarks on this paper.

\section{References}

[1] Agard, S. B., and F. W. Gehring: Angles and quasiconformal mappings. - Proc. London Math. Soc. 14a, 1965, 1-21.

[2] Aseev, V.V.: Quasisymmetric embeddings, complex analysis and representation theory. - J. Math. Sci. (New York) 108, 2002, 375-410.

[3] BAlogh, Z. M., and P. Koskela: Quasiconformality, quasisymmetry and removability in Loewner spaces. - Duke J. Math. 101, 2000, 554-577.

[4] Beurling, A., and L.V. Ahlfors: The boundary correspondence under quasiconformal mappings. - Acta Math. 96, 1956, 125-142.

[5] Bonk, M., and B. Kleiner: Rigidity for quasi-Möbius group actions. - J. Differential Geom. $61,2002,81-106$.

[6] Buckley, S. M., D. A. Herron, and X. XIE: Metric space inversions, quasihyperbolic distance, and uniform spaces. - Indiana Univ. Math. J. 57, 2008, 837-890.

[7] Haïsisnsky, P.: A sewing problem in metric spaces. - Ann. Acad. Sci. Fenn. Math. 34, 2009, $319-345$.

[8] Huang, X., and J. Liu: Quasihyperbolic metric and quasisymmetric mappings in metric spaces. - Trans. Amer. Math. Soc. 367, 2015, 6225-6246.

[9] Huang, M., S. Ponnusamy, A. Rasila, and X. Wang: On quasisymmetry of quasiconformal mappings. - Adv. Math. 288, 2016, 1069-1096.

[10] Tukia, P., and J. VäısÄLÄ: Quasisymmetric embeddings of metric spaces. - Ann. Acad. Sci. Fenn. Ser. A I Math. 5, 1980, 97-114.

[11] VÄısÄLÄ, J.: Quasimöbius maps. - J. Analyse Math. 44, 1984/1985, 218-234.

[12] VÄIsÄLÄ, J.: Free quasiconformality in Banach spaces. I. - Ann. Acad. Sci. Fenn. Ser. A I Math. 15, 1990, 355-379.

[13] VÄIsäLÄ, J.: Free quasiconformality in Banach spaces. II. - Ann. Acad. Sci. Fenn. Ser. A I Math. 16, 1991, 255-310.

[14] Wang, X., and Q. Zhou: Quasimöbius maps, weakly Quasimöbius maps and uniform perfectness in quasi-metric spaces. - Ann. Acad. Sci. Fenn. Math. 42, 2017, 257-284. 